\title{
LOCAL LIPSCHITZ CONTINUITY OF SOLUTIONS OF NON-LINEAR ELLIPTIC DIFFERENTIAL-FUNCTIONAL EQUATIONS
}

\author{
Pierre Bousquet ${ }^{1}$
}

\begin{abstract}
The object of this paper is to prove existence and regularity results for non-linear elliptic differential-functional equations of the form $\operatorname{div} a(\nabla u)+F[u](x)=0$, over the functions $u \in W^{1,1}(\Omega)$ that assume given boundary values $\phi$ on $\partial \Omega$. The vector field $a: \mathbb{R}^{n} \rightarrow \mathbb{R}^{n}$ satisfies an ellipticity condition and for a fixed $x, F[u](x)$ denotes a non-linear functional of $u$. In considering the same problem, Hartman and Stampacchia [Acta Math. 115 (1966) 271-310] have obtained existence results in the space of uniformly Lipschitz continuous functions when $\phi$ satisfies the classical bounded slope condition. In a variational context, Clarke [Ann. Sc. Norm. Super. Pisa Cl. Sci. 4 (2005) 511-530] has introduced a new type of hypothesis on the boundary condition $\phi$ : the lower (or upper) bounded slope condition. This condition, which is less restrictive than the previous one, is satisfied if $\phi$ is the restriction to $\partial \Omega$ of a convex function. We show that if $a$ and $F$ satisfy hypotheses similar to those of Hartman and Stampacchia, the lower bounded slope condition implies the existence of solutions in the space of locally Lipschitz continuous functions on $\Omega$.
\end{abstract}

Mathematics Subject Classification. 35J25, 35J60.

Received March 14, 2006.

Published online July 20, 2007.

\section{INTRODUCTION}

We study a Dirichlet boundary value problem associated with the following non-linear elliptic differentialfunctional equation:

$$
\operatorname{div}[a(\nabla u)]+F[u]=0 .
$$

We seek solutions in the space of functions $u \in W^{1,1}(\Omega)$ (where $\Omega$ is an open bounded convex set in $\mathbb{R}^{n}, n \geq 2$ ), whose trace $\operatorname{tr} u$ on $\Gamma:=\partial \Omega$ is equal to some function $\phi: \Gamma \rightarrow \mathbb{R}$. For a fixed $x \in \Omega, F[u](x)$ is a non-linear functional of $u$. For example, Hartman and Stampacchia consider the Euler equation of the variational problem

$$
\min \left\{\int_{\Omega} f(\nabla u) \mathrm{d} x-\left[\int_{\Omega} h(x, u) \mathrm{d} x\right]^{\beta}\right\}
$$

Keywords and phrases. Non-linear elliptic PDE's, Lipschitz continuous solutions, lower bounded slope condition.

${ }^{1}$ Institut Camille Jordan, Université Claude Bernard, Lyon 1, France; bousquet@math.univ-lyon1.fr

(c) EDP Sciences, SMAI 2007 
Then, $a_{j}(p)=f_{p_{j}}(p), F[u](x)=G[u] g(x, u)$ with

$$
G[u]=\left[\int_{\Omega} h(x, u(x))\right]^{\beta-1} \text { and } g(x, u)=\beta h_{u}(x, u) .
$$

We say that $u \in W^{1,1}(\Omega)$ is a weak solution of $(1.1)$ in $W_{\phi}^{1,1}(\Omega)$ (the space of functions in $W^{1,1}(\Omega)$ whose trace $\operatorname{tr} u$ is equal to the function $\phi)$ if $a(\nabla u) \in L_{\text {loc }}^{1}(\Omega), F[u] \in L_{\text {loc }}^{1}(\Omega)$ and

$$
\text { (E) } \int_{\Omega}\{\langle a(\nabla u(x)), \nabla \eta(x)\rangle-F[u](x) \eta(x)\} \mathrm{d} x=0
$$

for all continuously differentiable $\eta$ with compact support in $\Omega$; that is, $\eta \in C_{c}^{1}(\Omega)$.

The problem (E) has been tackled by Hartman and Stampacchia, among many others, in [6], which will be a recurrent reference throughout this paper. There, the authors show the existence of solutions to $(E)$ in the space $\operatorname{Lip}(\Omega, \phi)$ of uniformly Lipschitz continuous functions on $\bar{\Omega}$ whose trace on $\Gamma$ is $\phi$. Their proof is based on two main tools. The first one is an abstract existence theorem in functional analysis. This theorem enables them to assert for each $K>0$, the existence of a solution $u_{K}$ to $(E)$ in the space $\operatorname{Lip}(\Omega, \phi, K)$ of uniformly Lipschitz continuous functions of Lipschitz rank no greater than $K$. The second tool of the proof is an a priori bound on the Lipschitz rank of $u_{K}$, independently of $K$. Then, Hartman and Stampacchia obtain the desired solution $u$ in the space $\operatorname{Lip}(\Omega, \phi)$ as a limit, as $K \rightarrow \infty$, of the sequence $\left(u_{K}\right)$.

We are mainly interested in the generalization of the second tool: the a priori bound on the Lipschitz rank. In [6], it is based on a maximum principle on the gradient of the solutions, which can be stated as follows (see Lem. 10.0 in $[6])$ :

$$
\|\nabla u\|_{L^{\infty}(\Omega)} \leq \sup _{x \in \Gamma, y \in \Omega} \frac{|u(x)-u(y)|}{|x-y|}+C
$$

where $C$ is a constant depending on the data of the problem.

This maximum principle had already appeared in a variational context (see [10]) to give a proof of the Hilbert-Haar theorem. It is based on a device due to Rado which amounts to the comparison of a solution $u$ and a translated version of $u$, say $u_{\tau}:=u(\cdot+\tau)$ which is (nearly) a solution of the same equation but on $\Omega_{\tau}:=\Omega-\tau$.

To estimate the right hand side of (1.2), Hartman and Stampacchia consider the barrier technique.This technique has been widely used in the theory of elliptic pde's (see [4]). In particular, Lieberman (see [7-9]) has studied the relationship between the regularity of $\phi$ on $\Gamma$ and the regularity of the solutions on $\Omega$. Nevertheless, he always posits assumptions on the upper growth of $a$, which is not the case in our main result.

In [6], different types of hypotheses on $\phi$ are considered.One of them requires that $\phi$ satisfy the bounded slope condition (BSC). The BSC of rank $Q$ is the assumption that, given any point $\gamma \in \Gamma$, there exist two affine functions

$$
y \mapsto\left\langle\zeta_{\gamma}^{-}, y-\gamma\right\rangle+\phi(\gamma), y \mapsto\left\langle\zeta_{\gamma}^{+}, y-\gamma\right\rangle+\phi(\gamma)
$$

agreeing with $\phi$ at $\gamma$, whose slopes satisfy $\left|\zeta_{\gamma}^{-}\right| \leq Q,\left|\zeta_{\gamma}^{+}\right| \leq Q$, and such that

$$
\left\langle\zeta_{\gamma}^{-}, \gamma^{\prime}-\gamma\right\rangle+\phi(\gamma) \leq \phi\left(\gamma^{\prime}\right) \leq\left\langle\zeta_{\gamma}^{+}, \gamma^{\prime}-\gamma\right\rangle+\phi(\gamma), \forall \gamma^{\prime} \in \Gamma .
$$

This condition forces $\phi$ to be affine on 'flat parts' of $\Gamma$. Moreover, if $\Omega$ is smooth, then it forces $\phi$ to be smooth as well (see Hartman [5] for precise statements; see also [1]).

Recently, Clarke [3] has introduced a new hypothesis on $\phi$, the lower bounded slope condition (LBSC) of rank $Q$ : given any point $\gamma \in \Gamma$, there exists an affine function

$$
y \mapsto\left\langle\zeta_{\gamma}, y-\gamma\right\rangle+\phi(\gamma),
$$


with $\left|\zeta_{\gamma}\right| \leq Q$ such that

$$
\left\langle\zeta_{\gamma}, \gamma^{\prime}-\gamma\right\rangle+\phi(\gamma) \leq \phi\left(\gamma^{\prime}\right), \forall \gamma^{\prime} \in \Gamma
$$

This requirement enlarges the class of boundary functions which it allows, compared to the BSC. It can be shown in particular that $\phi: \Gamma \rightarrow \mathbb{R}$ satisfies the LBSC if and only if it is the restriction to $\Gamma$ of a convex function. When $\Omega$ is uniformly convex, $\phi$ satisfies the LBSC if and only if it is the restriction to $\Gamma$ of a semiconvex function (see [1] for details and further properties).

Clarke has shown in a variational context that the LBSC gives the local Lipschitz continuity of minimizers (see [3], see also [2]). The proof rests on a modification of Rado's device: The minimizer $u$ is compared now to a dilated version of $u$ (and not to a translated one).

The goal of this paper is to adapt the ideas appearing in [3] and [2], used in a variational context, to our present setting, so as to prove existence and local Lipschitz regularity of the solutions to the elliptic differentialfunctional equations considered above, when the LBSC is satisfied (rather than the BSC). We remark that local Lipschitzness is the crucial property to show further regularity results with the help of the De Giorgi's theory, when the data are regular enough (see [6], Sect. 14). In our context, however, we can only get local regularity; that is, on any compact subsets of $\Omega$.

The next section describes the hypotheses that we posit on the data, and the proof of our theorem is given in Section 3. The final section discusses the issue of the continuity of the solution at the boundary.

\section{The Main Result}

Recall that $\operatorname{Lip}(\Omega)$ denotes the set of uniformly Lipschitz continuous functions on $\Omega$ (or, equivalently, on $\bar{\Omega}$ ). Let $\operatorname{Lip}(\Omega, \phi)$ be the set of functions $u \in \operatorname{Lip}(\Omega)$ for which $u=\phi$ on $\Gamma$. For a given $K$, let $\operatorname{Lip}(\Omega, \phi, K)$ be the set of functions $u \in \operatorname{Lip}(\Omega, \phi)$ of rank $\leq K$ (this set being empty if $\phi$ is not Lipschitz of rank at most $K$ ). We now specify the hypotheses on the data of the problem $(E)$. Recall that

$(H \Omega) \quad \Omega$ is an open bounded convex set in $\mathbb{R}^{n}, n \geq 2$.

$(H \phi) \quad \phi$ satisfies the lower bounded slope condition of rank $Q$.

This implies that $\phi$ can be extended as a convex function on $\mathbb{R}^{n}$, which will be done henceforth. Moreover, we may assume that $\phi$ is globally Lipschitz of rank $Q$. As $\Omega$ is convex, it has a Lipschitz boundary, which justifies the use of trace in the boundary condition: $\operatorname{tr} u=\phi$.

We will assume that $a=\left(a_{1}, . ., a_{n}\right)$ is continuous on $\mathbb{R}^{n}$ and satisfies

$$
\langle a(p)-a(q), p-q\rangle \geq \mu_{0}|p-q|^{2},
$$

for some $\mu_{0}>0$. This implies (with $q=0$ ) that for any $\epsilon>0$, there exists $N_{\epsilon} \geq 0$ such that $\langle a(p), p\rangle \geq$ $\left(\mu_{0}-\epsilon\right)|p|^{2}-N_{\epsilon}$.

The non-linear functional $F$ satisfies the four hypotheses below (where $u$ is any bounded and continuous function on $\Omega$ ):

$(H F 0) \quad x \in \Omega \mapsto F[u](x)$ is well-defined and measurable,

$$
(H F 1) \quad F[u](x) \operatorname{sgn} u(x) \leq \sum_{i=1}^{m} c_{i}\|u\|_{L^{\alpha(i)}(\Omega)}^{\beta(i)}|u(x)|^{\gamma(i)-1}, x \in \Omega \text { a.e. }
$$

where $c_{i} \geq 0, \alpha(i) \geq 1, \beta(i) \geq 0, \gamma(i) \geq 1$ and $\alpha(i) \leq 2^{*}, \beta(i)+\gamma(i) \leq 2$. (As usual, $1 / 2^{*}=1 / 2-1 / n$ when $n>2$. If $n=2,2^{*}$ denotes any number larger than 4$)$. We also assume that the coefficients $c_{i}$ in $(H F 1)$ satisfy

$$
\mu_{0}-\sum{ }^{\prime} c_{i} \Lambda^{-2}|\Omega|^{1-2 / \sigma+\beta(i) / \alpha(i)}>0
$$


where $\sum^{\prime}$ is the sum over the indices $i$ for which $\beta(i)+\gamma(i)=2$. Here,

$$
\sigma:=\max _{i=1, . ., m}(\alpha(i), 2) \leq 2^{*}
$$

and

$$
\Lambda:=\inf _{u \in W_{0}^{1,2}(\Omega)} \frac{\|\nabla u\|_{L^{2}(\Omega)}}{\|u\|_{L^{\sigma}(\Omega)}} .
$$

Furthermore, we assume that for every number $M>0$, there exists a number $\chi(M)$ such that

$$
(H F 2) \quad|u(x)| \leq M \quad \text { on } \Omega \Rightarrow|F[u](x)| \leq \chi(M) .
$$

The last hypothesis on $F$ is:

(HF3) If $u_{h} \in \operatorname{Lip}(\Omega, \phi)$ for $h=1,2, \ldots$ is a bounded sequence

in $L^{\infty}(\Omega)$ which converges to $u$ uniformly on compact subsets

of $\Omega$ as $h \rightarrow \infty$, then $F\left[u_{h}\right](x) \rightarrow F[u](x)$ a.e. on $\Omega$.

These hypotheses are closely related to those of Hartman and Stampacchia [6]. They are satisfied by the example given in the introduction.

We can pick some $\epsilon>0$ such that inequality (2.3) remains true when $\mu_{0}$ is replaced by $\mu:=\mu_{0}-\epsilon>0$. With that $\mu,(H a)$ remains true and we have

$$
\langle a(p), p\rangle \geq \mu|p|^{2}-N
$$

for some $N>0$.

Under these hypotheses, we can state our theorem:

Theorem 2.1. Under hypotheses $(H \Omega),(H \phi),(H a)$ and $(H F 0),(H F 1),(H F 2),(H F 3)$, there exists a locally Lipschitz $u \in W_{\phi}^{1,2}(\Omega) \cap L^{\infty}(\Omega)$ which satisfies $(E)$ :

$$
\int_{\Omega}\{\langle a(\nabla u(x)), \nabla \eta(x)\rangle-F[u](x) \eta(x)\} \mathrm{d} x=0 \quad \forall \eta \in C_{c}^{1}(\Omega) .
$$

This theorem generalises Theorem 12.1 in the article of Hartman and Stampacchia [6], in the sense that the bounded slope condition is reduced to the lower bounded slope condition. In contrast to [6], however, we do not assert the global Lipschitzness of the solution. This explains why the hypotheses that we have made on $a$ and $F$ are more restrictive than those appearing in [6]. In particular, a small dependence on the gradient is allowed there in the hypothesis corresponding to $(H F 2)$.

In fact, it is not the case in our context that solutions are globally Lipschitz, as evidenced by the following example (see $[1,3])$ :

Example 2.1. The set $\Omega$ is the open disc in $\mathbb{R}^{2}, \phi(\cos \theta, \sin \theta):=-\pi^{2} / 6+\pi / 2 \theta-\theta^{2} / 4,0 \leq \theta<2 \pi, F=0$ and $a(p)=p$. Then the solution of $(E)$ is locally Lipschitz but not globally Lipschitz.

Remark 2.1. There is another version of the theorem where the lower bounded slope condition is replaced by an upper bounded slope condition. 


\section{Proof of the theorem}

Following the terminology of [6], by a $K$ quasi solution of (1.1) will be meant a function $u \in \operatorname{Lip}(\Omega, \phi, K)$ satisfying

$$
\int_{\Omega}\{\langle a(\nabla u), \nabla(v-u)\rangle-F[u](v-u)\} \geq 0, \quad \forall v \in \operatorname{Lip}(\Omega, \phi, K) .
$$

We recall here some results of [6]. First, the following existence theorem holds (this is [6], Lem. 12.1).

Proposition 3.1. For every $K>Q$, there exists a $K$ quasi solution to (3.5).

The following proposition (which is exactly Th. 8.1 in [6]) provides an a priori bound in $L^{\infty}(\Omega)$ for any $K$ quasi solution $(K>Q)$.

Proposition 3.2. There exists a constant $T$ (independent of $K$ ) such that if $u$ is a $K$ quasi solution of (3.5), then

$$
|u(x)| \leq T, \quad \text { on } \Omega
$$

From this bound, we can infer easily an a priori bound in $W^{1,2}(\Omega)$ :

Proposition 3.3. There exists a constant $T^{\prime}$ (independent of $K>Q$ ) such that if $u$ is a $K$ quasi solution of (3.5), then

$$
\|u\|_{W^{1,2}} \leq T^{\prime}
$$

Proof. Since $\phi \in \operatorname{Lip}(\Omega, \phi, K)$, we have

$$
\int_{\Omega}\left\langle a\left(\nabla u_{K}\right), \nabla\left(u_{K}-\phi\right)\right\rangle \leq \int_{\Omega} F\left[u_{K}\right]\left(u_{K}-\phi\right)
$$

so that

$$
\int_{\Omega}\left\langle a(\nabla \phi), \nabla\left(u_{K}-\phi\right)\right\rangle+\mu \int_{\Omega}\left|\nabla\left(u_{K}-\phi\right)\right|^{2} \leq \int_{\Omega} F\left[u_{K}\right]\left(u_{K}-\phi\right) .
$$

Then (using the fact that $\left\|u_{K}-\phi\right\|_{L^{\infty}(\Omega)} \leq T+\|\phi\|_{L^{\infty}(\Omega)}$ ),

$$
\mu \int_{\Omega}\left|\nabla\left(u_{K}-\phi\right)\right|^{2} \leq\|a(\nabla \phi)\|_{L^{\infty}(\Omega)} \int_{\Omega}\left|\nabla\left(u_{K}-\phi\right)\right|+\chi(T)\left(T+\|\phi\|_{L^{\infty}(\Omega)}\right)
$$

where $\chi(T)$ is given by $(H F 2)$. Writing that

$$
\left\|a(\nabla \phi)||_{L^{\infty}(\Omega)} \int_{\Omega}\left|\nabla\left(u_{K}-\phi\right)\right| \leq \epsilon \int_{\Omega}\left|\nabla\left(u_{K}-\phi\right)\right|^{2}+\right\| a(\nabla \phi) \|_{L^{\infty}(\Omega)}^{2}|\Omega| /(4 \epsilon)
$$

we see that $\left\|\nabla\left(u_{K}-\phi\right)\right\|_{L^{2}(\Omega)}$ is bounded by a constant depending on $\|a(\nabla \phi)\|_{L^{\infty}(\Omega)}, \mu, T,\|\phi\|_{L^{\infty}(\Omega)}$ and $\Omega$. Hence, $\left(u_{K}\right)$ is bounded in $W^{1,2}(\Omega)$. This completes the proof.

The proof of Theorem 2.1 uses the well-known barrier technique:

Proposition 3.4. There exists $\bar{Q} \geq 0$ such that for any $\gamma \in \Gamma$, there exists $w: \bar{\Omega} \rightarrow \mathbb{R}$ Lipschitz of rank $\bar{Q}$ which satisfies

$$
w(\gamma)=\phi(\gamma), w(y) \leq u(y) \quad \forall y \in \Omega
$$

for any $K$ quasi solution $u$ of (3.5) and any $K>\bar{Q}$. 
Proof. We build the same barrier as in [2], Theorem 2.2. There is an element $\zeta$ with $|\zeta| \leq Q$ in the subdifferential of $\phi$ at $\gamma$ :

$$
\phi(x)-\phi(\gamma) \geq\langle\zeta, x-\gamma\rangle \quad \forall x \in \mathbb{R}^{n} .
$$

By (HF2) and Proposition 3.2, $|F[u](x)| \leq \chi(T) x \in \Omega$ a.e., for any $K$ quasi-solution $u$ of (3.5). Fix any $R>(\chi(T)+1) \exp (\operatorname{diam} \Omega) / \mu$ where $\mu$ is given by $(2.4)$. Recall that $(H a)$ remains true when $\mu_{0}$ is replaced by $\mu$. Let $\nu$ be a unit outward normal vector to $\bar{\Omega}$ at $\gamma$ and define

$$
w(x):=\phi(\gamma)+\langle\zeta, x-\gamma\rangle-R\{1-\exp (\langle x-\gamma, \nu\rangle)\} .
$$

The function $w$ agrees with $\phi$ at $\gamma$ and is Lipschitz of rank

$$
\bar{Q}:=Q+R \exp (\operatorname{diam} \Omega) .
$$

Let $K>\bar{Q}$ and $u$ be a $K$ quasi solution of (3.5). We have to show that the set

$$
S:=\{x \in \Omega: w(x)>u(x)\}
$$

has measure 0 . The function $M(x):=\max [u(x), w(x)]$ is Lipschitz of rank $K$ and its trace is $\phi$ (this follows from the subgradient inequality for $\zeta$ and the fact that $\langle x-\gamma, \nu\rangle \leq 0$ for $x \in \Omega$ ).

As $u$ is a $K$ quasi solution of (3.5) (relative to $M$ ), we have

$$
\int_{S}\langle a(\nabla u), \nabla(u-w)\rangle \leq \int_{S} F[u](u-w) .
$$

Thanks to $(H a)$, we get

$$
\int_{S}\langle a(\nabla w), \nabla(u-w)\rangle \leq \chi(T) \int_{S}(w-u)
$$

Let us make the temporary assumption that $a$ is $C^{1}$. Then, a straightforward calculation yields

$$
\begin{aligned}
\operatorname{div}[a(\nabla w)] & =R \exp (\langle x-\gamma, \nu\rangle) \sum_{i, j} \partial_{p_{j}} a_{i}(\nabla w) \nu_{i} \nu_{j} \\
& \geq \chi(T)+1
\end{aligned}
$$

in light of (Ha) and because of how $R$ was chosen. Then, (3.7) implies:

$$
\begin{aligned}
\chi(T) \int_{S}(w-u) & \geq \int_{S}\langle a(\nabla w), \nabla(u-w)\rangle \\
& \geq \int_{S}(w-u) \operatorname{div}[a(\nabla w)] \\
& \geq(\chi(T)+1) \int_{S}(w-u) .
\end{aligned}
$$

This shows that $S$ is of measure 0 , since $w-u>0$ on $S$.

In the general case in which $a$ is not $C^{1}$, we consider a sequence $a_{k}$ of $C^{1}$ vector fields converging to $a$ uniformly on compact sets and satisfying $(\mathrm{Ha})$. Then, for each $k$,

$$
\int_{S}\left\langle a_{k}(\nabla w), \nabla(u-w)\right\rangle \geq(\chi(T)+1) \int_{S}(w-u)
$$

and the quantity $\int_{S}\left\langle a_{k}(\nabla w), \nabla(u-w)\right\rangle$ converges to $\int_{S}\langle a(\nabla w), \nabla(u-w)\rangle$ as $k$ goes to $+\infty$. This shows that the result is still true when $a$ is merely assumed continuous. 
We then proceed exactly as in [2]. Consider a $K$ quasi solution $u$ of (3.5). Let $\lambda \in[1 / 2,1), q>\bar{q}:=$ $\bar{Q} \operatorname{diam} \Omega+\|\phi\|_{L^{\infty}(\Omega)}$ and $z \in \Gamma$. We will denote by

$$
\begin{aligned}
u_{\lambda}(x) & :=\lambda u((x-z) / \lambda+z)-q(1-\lambda) \\
\Omega_{\lambda} & :=\lambda(\Omega-z)+z .
\end{aligned}
$$

Then $u_{\lambda}$ belongs to the space $\operatorname{Lip}\left(\Omega_{\lambda}, \phi_{\lambda}, K\right)$, where $\phi_{\lambda}(x):=\lambda \phi((x-z) / \lambda+z)-q(1-\lambda)$. We want to compare $u_{\lambda}$ and $u$ on $\Gamma_{\lambda}:=\partial \Omega_{\lambda}$.

This is done by the following proposition, whose proof appears in [2]:

Proposition 3.5. We have $u_{\lambda} \leq u$ on $\Gamma_{\lambda}$.

The next step of the proof is to show that the set

$$
A:=\left\{y \in \Omega_{\lambda}: u_{\lambda}(y)>u(y)\right\}
$$

has measure zero. Once again, the proof is very similar to that of [2]:

By definition of a $K$ quasi solution, $u \in \operatorname{Lip}(\Omega, \phi, K)$ satisfies (3.5); that is,

$$
\int_{\Omega}\langle a(\nabla u), \nabla(v-u)\rangle-F[u](v-u) \geq 0, \quad \forall v \in \operatorname{Lip}(\Omega, \phi, K) .
$$

We will denote $F[u](x)$ by $g(x)$ for all $x \in \Omega$. Then, $g \in L^{\infty}(\Omega)$.

Let $w(x):=\min \left(u_{\mid \Omega_{\lambda}}, u_{\lambda}\right) \in \operatorname{Lip}\left(\Omega_{\lambda}, \phi_{\lambda}, K\right)$ (thanks to Prop. 3.5).

Let $w^{\lambda}(x):=1 / \lambda w(\lambda(x-z)+z)+q(1 / \lambda-1) \in \operatorname{Lip}(\Omega, \phi, K)$. With $v:=w^{\lambda}$ in (3.5), we get after an obvious change of variables

$$
0 \leq \int_{\Omega_{\lambda}}\left\langle a\left(\nabla u_{\lambda}(y)\right), \nabla w(y)-\nabla u_{\lambda}(y)\right\rangle-g\left(\frac{y-z}{\lambda}+z\right)\left(\frac{w}{\lambda}(y)-\frac{u_{\lambda}}{\lambda}(y)\right) \mathrm{d} y
$$

which implies

$$
0 \leq \int_{A}\left\langle a\left(\nabla u_{\lambda}(y)\right), \nabla u(y)-\nabla u_{\lambda}(y)\right\rangle-\frac{1}{\lambda} g\left(\frac{y-z}{\lambda}+z\right)\left(u(y)-u_{\lambda}(y)\right) \mathrm{d} y .
$$

Let $W(x):=\max \left(u_{\mid \Omega_{\lambda}}(x), u_{\lambda}(x)\right)$ for $x \in \Omega_{\lambda}$ and $W(x):=u(x)$ for $x \in \Omega-\Omega_{\lambda}$. Then $W \in \operatorname{Lip}(\Omega, \phi, K)$. With $v:=W$ in $(3.5)$, we get

$$
0 \leq \int_{A}\left\langle a(\nabla u), \nabla\left(u_{\lambda}-u\right)\right\rangle-g(y)\left(u_{\lambda}-u\right) .
$$

Summing these two last inequalities, we get

$$
0 \leq \int_{A}\left\langle-a\left(\nabla u_{\lambda}\right)+a(\nabla u), \nabla\left(u_{\lambda}-u\right)\right\rangle+\left(\frac{1}{\lambda} g\left(\frac{y-z}{\lambda}+z\right)-g(y)\right)\left(u_{\lambda}-u\right)
$$

so that using $(H a)$,

$$
\mu \int_{A}\left|\nabla\left(u_{\lambda}-u\right)\right|^{2} \leq \int_{A}\left(\frac{1}{\lambda} g\left(\frac{y-z}{\lambda}+z\right)-g(y)\right)\left(u_{\lambda}-u\right) .
$$

We proceed with the following lemma (the proof of which can be found in [2]; see the calculations following inequality (6) there):

Lemma 3.1. Let $u \in W^{1,2}(\Omega), g \in L^{\infty}(\Omega)$ and $\mu>0$. Assume that there exists $\bar{q}$ such that for any $q>\bar{q}$ and $\lambda \in[1 / 2,1)$, we have

$$
\mu \int_{A}\left|\nabla\left(u_{\lambda}-u\right)\right|^{2} \leq \int_{A}\left(\frac{1}{\lambda} g\left(\frac{y-z}{\lambda}+z\right)-g(y)\right)\left(u_{\lambda}-u\right),
$$


where $u_{\lambda}, \Omega_{\lambda}$ are defined as in (3.8), (3.9) and $A=A(q):=\left\{y \in \Omega_{\lambda}: u_{\lambda}(y)>u(y)\right\}$. Then, there exists $q_{0}>\bar{q}$ such that $A(q)$ has measure 0 for any $q \geq q_{0}$. The number $q_{0}$ only depends on $\bar{q}, n, \mu, \Omega,\|g\|_{L^{\infty}(\Omega)}$ but not on $u, \lambda \in[1 / 2,1)$ nor on $z \in \Gamma$.

We infer from this lemma that there exists $q_{0}>0$ such that

$$
\lambda u((x-z) / \lambda+z) \leq u(x)+q(1-\lambda) \quad \forall q \geq q_{0} .
$$

This implies that the Lipschitz rank of $u$ can be bounded independently of $K$ on any compact set of $\Omega$, as shown by the following lemma (for a proof of this one, see the final step of the proof of the main theorem in [2]):

Lemma 3.2. Let $u \in L^{\infty}(\Omega)$. Assume that there exists $q_{0}>0$ such that for any $\lambda \in[1 / 2,1), z \in \Gamma$, we have:

$$
\lambda u((y-z) / \lambda+z)-q_{0}(1-\lambda) \leq u(y),
$$

a.e. $y \in \Omega_{\lambda}:=\lambda(\Omega-z)+z$. Then, $u$ (admits a representative which) is locally Lipschitz on $\Omega$ and we have

$$
|D u(x)| \leq \frac{\|u\|_{L^{\infty}(\Omega)}+q_{0}}{d_{\Gamma}(x)}, \quad x \in \Omega \text { a.e. }
$$

where $d_{\Gamma}$ denotes the distance to $\Gamma$.

We may summarize the current state of the proof as follows: for each $K>0$, there exists $u_{K} \in \operatorname{Lip}(\Omega, \phi, K)$ a $K$ quasi solution of (3.5), such that $\left\|u_{K}\right\|_{L^{\infty}(\Omega)} \leq T,\left\|u_{K}\right\|_{W^{1,2}}(\Omega) \leq T^{\prime}$ and the Lipschitz rank of $u_{K}$ on any compact subset $\Omega_{0} \subset \Omega$ is bounded by

$$
\frac{T+q_{0}}{\mathrm{~d}\left(\Omega_{0}, \Gamma\right)}
$$

where $q_{0}, T$ are independent of $K$ and d $\left(\Omega_{0}, \Gamma\right)$ denotes the distance between $\Omega_{0}$ and $\Gamma$. Let $\Omega_{j}$ be an increasing sequence of open subsets of $\Omega$ satisfying $\Omega_{j} \subset \bar{\Omega}_{j} \subset \Omega_{j+1}$ and $\cup_{j \geq 1} \Omega_{j}=\Omega$. Let $K_{j}$ be a common Lipschitz rank for all the functions $u_{K}$ restricted to $\Omega_{j}$. Then, up to a subsequence, the functions $u_{K}$ converge uniformly on every compact subset of $\Omega$ to a function $u$ which is Lipschitz of rank $K_{j}$ on $\Omega_{j}$. Moreover, we can suppose that for every $j, \nabla u_{K}$ converges to $\nabla u$ in $\sigma\left(L^{\infty}\left(\Omega_{j}\right), L^{1}\left(\Omega_{j}\right)\right)$. Finally, we can also assume that $\left(u_{K}\right)$ converges weakly to $u$ in $W^{1,2}(\Omega)$. It remains to show that:

Proposition 3.6. The function $u$ is a weak solution of $(E)$ and $u \in W_{0}^{1,2}(\Omega)+\phi$.

Proof. This second assertion is trivial in view of the weak convergence in $W^{1,2}(\Omega)$. Fix some $\eta \in C_{c}^{\infty}(\Omega)$. Let $L$ be a Lipschitz constant for $\eta$. Let $\epsilon>0$. We know that $\left\|F\left[u_{K}\right]\right\|_{L^{\infty}} \leq \chi(T)$. Let $j$ be big enough so that $\operatorname{supp} \eta \subset \Omega_{j}$. Let $\epsilon_{j}$ be such that

$$
\epsilon_{j}\left(L+3 K_{j+1}+1\right) \sup _{|p| \leq L+3 K_{j+1}+1}|a(p)| \leq \epsilon
$$

and such that

$$
\epsilon_{j}\left(\chi(T)\left(\|\eta\|_{L^{\infty}(\Omega)}+2 T\right)\right)<\epsilon .
$$

Let $\Omega_{j}^{\prime}$ be an open subset of $\Omega$ such that

$$
\bar{\Omega}_{j} \subset \Omega_{j}^{\prime} \subset \bar{\Omega}_{j}^{\prime} \subset \Omega_{j+1}
$$

and $\left|\Omega_{j}^{\prime} / \Omega_{j}\right|<\epsilon_{j}$.

Let $\theta_{j} \in C_{c}^{\infty}\left(\Omega_{j}^{\prime}\right)$ such that $\theta_{j} \equiv 1$ on $\Omega_{j}$ and $0 \leq \theta_{j} \leq 1$.

Let

$$
\psi_{K}(x):=u_{K}(x)+\eta(x)+\theta_{j}(x)\left(u(x)-u_{K}(x)\right) .
$$


For any $K \geq 0, \psi_{K}(x)=u_{K}(x)$ on $\Omega / \Omega_{j}^{\prime}$ and $\psi_{K}(x)=\eta(x)+u(x)$ on $\Omega_{j}$. A Lipschitz rank for $\psi_{K}$ restricted to $\Omega_{j}^{\prime}$ is $S_{j}:=K_{j+1}+L+2 T|| \nabla \theta_{j} \|_{L^{\infty}\left(\Omega_{j}^{\prime}\right)}$. Then, for any $K \geq S_{j}, \psi_{K}$ is $K$ Lipschitz on $\Omega$ and we have:

$$
\int_{\Omega}\left\langle a\left(\nabla u_{K}\right), \nabla\left(\psi_{K}-u_{K}\right)\right\rangle \geq \int_{\Omega} F\left[u_{K}\right](x)\left(\psi_{K}-u_{K}\right)(x) \mathrm{d} x
$$

which implies

$$
\int_{\Omega_{j}^{\prime}}\left\langle a\left(\nabla \psi_{K}\right), \nabla\left(\psi_{K}-u_{K}\right)\right\rangle \geq \int_{\Omega_{j}^{\prime}} F\left[u_{K}\right](x)\left(\psi_{K}-u_{K}\right)(x) \mathrm{d} x
$$

Hence,

$$
\int_{\Omega_{j}}\left\langle a(\nabla(\eta+u)), \nabla\left(\eta+u-u_{K}\right)\right\rangle \geq \int_{\Omega_{j}} F\left[u_{K}\right](x)\left(\eta+\theta_{j}\left(u-u_{K}\right)\right)(x) \mathrm{d} x-2 \epsilon .
$$

Passing to the limit when $K \rightarrow+\infty$ yields

$$
\int_{\Omega_{j}}\langle a(\nabla(\eta+u)), \nabla \eta\rangle \geq \int_{\Omega_{j}} F[u](x) \eta(x) \mathrm{d} x-2 \epsilon .
$$

(recall that $\nabla u \rightarrow \nabla u_{K}$ in $\sigma\left(L^{\infty}, L^{1}\right)$ and $F\left[u_{K}\right](x) \rightarrow F[u](x)$ a.e. and is bounded independently of $K$.) In the previous inequality, we can replace $\Omega_{j}$ by $\Omega$ (since supp $\eta \subset \Omega_{j}$ ) and notice that $\epsilon$ is arbitrary. We have then shown that

$$
\int_{\Omega}\langle a(\nabla(\eta+u)), \nabla \eta\rangle \geq \int_{\Omega} F[u](x) \eta(x) \mathrm{d} x .
$$

Replace now $\eta$ by $t \eta$ for any $t \in \mathbb{R} \backslash\{0\}$, divide by $t$ and let $t \rightarrow 0$. Then

$$
\int_{\Omega}\langle a(\nabla u), \nabla \eta\rangle=\int_{\Omega} F[u](x) \eta(x) \mathrm{d} x .
$$

This shows that $u$ is a weak solution of $(E)$ and completes the proof of the theorem.

\section{Continuity at the Boundary}

We know by example that in general, $u$ fails to be globally Lipschitz. But there remains the question of whether $u$ is continuous at the boundary. Under merely the hypotheses of Theorem 2.1, it is an open problem. However, the continuity on $\bar{\Omega}$ can be proved under additional hypotheses on $\Omega$ and/or on the integrability of $u$.

This conclusion is based on the following properties of $u$. First, there exists a function $\bar{w} \in \operatorname{Lip}(\Omega, \phi, \bar{Q})$ (for some $\bar{Q}>0$ ) such that $w \leq u$ on $\Omega$. Indeed, if we denote by $w_{\gamma}$ the function built in Proposition 3.4, then the function $\bar{w}:=\inf _{\gamma \in \Gamma} w_{\gamma}$ belongs to $\operatorname{Lip}(\Omega, \phi, \bar{Q})$ and satisfies:

$$
w_{\gamma} \leq u_{K}, \quad \forall K>\bar{Q} .
$$

So, the same inequality is satisfied by $u$ instead of $u_{K}$.

Secondly, inequality (3.10) easily implies

$$
u(y) \leq u(x)+\left(q_{0}+\|\left. u\right|_{L^{\infty}(\Omega)}\right) \frac{|x-y|}{|y-z|}
$$

whenever $|y-x|<1 / 2|y-z|$, with $z \in \Gamma$ such that $y=(x-z) / \lambda+z$ for some $\lambda \in(1 / 2,1)$.

The arguments of [3] (namely, the proofs of Ths. 2.2, 2.3 and 2.4) show the following: 
Theorem 4.1. If $\Gamma$ is a polyhedron, then $u$ is Hölder continuous on $\bar{\Omega}$ of order $1 /(n+2)$. If $\Gamma$ is $C^{1,1}$ and $u \in W^{1, p}$ with $p>(n+1) / 2$, then $u$ satisfies on $\bar{\Omega}$ a Hölder condition of order

$$
a:=\frac{2 p-n-1}{4 p+n-3}
$$

Acknowledgements. The author thanks Francis Clarke for many helpful remarks.

\section{REFERENCES}

[1] P. Bousquet, The lower bounded slope condition. J. Convex Anal. 14 (2007) 119-136.

[2] P. Bousquet and F. Clarke, Local Lipschitz continuity of solutions to a problem in the calculus of variations. J. Differ. Eq. (to appear).

[3] F. Clarke, Continuity of solutions to a basic problem in the calculus of variations. Ann. Sc. Norm. Super. Pisa Cl. Sci. 4 (2005) $511-530$.

[4] D. Gilbarg and N.S. Trudinger, Elliptic partial differential equations of second order. Classics in Mathematics, Springer-Verlag, Berlin (2001). Reprint of the 1998 edition.

[5] P. Hartman, On the bounded slope condition. Pacific J. Math. 18 (1966) 495-511.

[6] P. Hartman and G. Stampacchia, On some non-linear elliptic differential-functional equations. Acta Math. 115 (1966) $271-310$.

[7] G.M. Lieberman, The quasilinear Dirichlet problem with decreased regularity at the boundary. Comm. Partial Differential Equations 6 (1981) 437-497.

[8] G.M. Lieberman, The Dirichlet problem for quasilinear elliptic equations with Hölder continuous boundary values. Arch. Rational Mech. Anal. 79 (1982) 305-323.

[9] G.M. Lieberman, The Dirichlet problem for quasilinear elliptic equations with continuously differentiable boundary data. Comm. Partial Differential Equations 11 (1986) 167-229.

[10] M. Miranda, Un teorema di esistenza e unicità per il problema dell'area minima in $n$ variabili. Ann. Scuola Norm. Sup. Pisa 19 (1965) 233-249. 